Erratum

\title{
Erratum: Up-regulation of LINC00161 correlates with tumor migration and invasion and poor prognosis of patients with hepatocellular carcinoma
}

\author{
Li-Chao Xu ${ }^{1, *}$, Quan-Ning Chen ${ }^{3, *}$, Xi-Qiang Liu ${ }^{4, *}$, Xiao-Ming Wang ${ }^{5}$, Qi-Meng \\ Chang ${ }^{6}$, Qi Pan ${ }^{2}$, Lu Wang ${ }^{2}$ and Yi-Lin Wang ${ }^{2}$ \\ ${ }^{1}$ Department of Interventional Radiology, Fudan University Shanghai Cancer Center, Department of Oncology, Shanghai \\ Medical College, Fudan University, Shanghai, 200032, China \\ 2 Department of Hepatic Surgery, Fudan University Shanghai Cancer Center, Department of Oncology, Shanghai Medical \\ College, Fudan University, Shanghai, 200032, China \\ ${ }^{3}$ Department of General Surgery, Tongji Hospital, School of Medicine, Tongji University, Shanghai, 200065, China \\ ${ }^{4}$ Department of Hepatobiliary-Pancreatic Surgery, Hangzhou, 310014, China \\ ${ }^{5}$ Department of Hepatobiliary Surgery, Yi Ji-shan Hospital, Wan Nan Medical College, Wuhu, 241000, China \\ ${ }^{6}$ Department of General Surgery, Minhang Hospital, Fudan University, Shanghai, 201199, China \\ \# These authors contributed equally to this work \\ Published: February 15, 2019
}

Copyright: Xu et al. This is an open-access article distributed under the terms of the Creative Commons Attribution License 3.0 (CC BY 3.0), which permits unrestricted use, distribution, and reproduction in any medium, provided the original author and source are credited.

This article has been corrected: During production, the affiliations for this article were listed incorrectly. The proper affiliations are shown below.

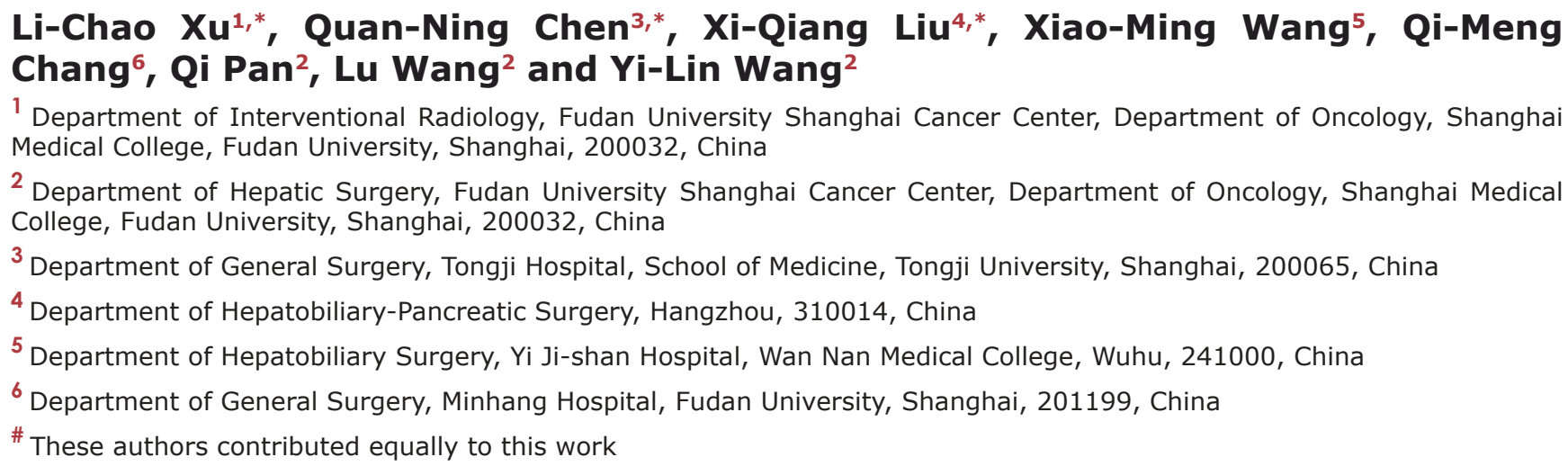

${ }^{1}$ Department of Interventional Radiology, Fudan University Shanghai Cancer Center, Department of Oncology, Shanghai Medical College, Fudan University, Shanghai, 200032, China

2 Department of Hepatic Surgery, Fudan University Shanghai Cancer Center, Department of Oncology, Shanghai Medical College, Fudan University, Shanghai, 200032, China

${ }^{3}$ Department of General Surgery, Tongji Hospital, School of Medicine, Tongji University, Shanghai, 200065, China

${ }^{4}$ Department of Hepatobiliary-Pancreatic Surgery, Hangzhou, 310014, China

${ }^{5}$ Department of Hepatobiliary Surgery, Yi Ji-shan Hospital, Wan Nan Medical College, Wuhu, 241000, China

${ }^{6}$ Department of General Surgery, Minhang Hospital, Fudan University, Shanghai, 201199, China

\# These authors contributed equally to this work

Original article: Oncotarget. 2017; 8:56168-56173. https://doi.org/10.18632/oncotarget.17040 